\title{
Larvas de Geometridae (Lepidoptera) e seus parasitóides, associadas a Erythroxylum microphyllum St.- Hilaire (Erythroxylaceae)
}

\author{
Gláucia Marconatoํㅜ, Manoel Martins Dias² \& Angélica Maria Penteado-Dias²
}

${ }^{1}$ Programa de Pós-graduação em Ecologia e Recursos Naturais. Universidade Federal de São Carlos. Caixa Postal 676. 13565-905 São Carlos-SP, Brasil.glauciam@usp.br

${ }^{2}$ Departamento de Ecologia e Biologia Evolutiva, Universidade Federal de São Carlos. manoelmd@power.ufscar.br; angelica@power.ufscar.br

\begin{abstract}
Geometrid larvae (Lepidoptera) and their parasitoids, associated to Erythroxylum microphyllum St.Hilaire (Erythroxylaceae). This work was carried out in a cerrado area, in São Carlos, São Paulo, Brazil. The samples were collected at each two weeks, from June/1999 to June/2000, with entomological umbrella. 22 species of Geometridae arranged in 14 genera were found; concerning parasitoids, 11 genera of Hymenoptera and two genera of Diptera, Tachinidae. The occurrence of Cyclomia mopsaria (Geometridae) was 83\%. Other Geometridae recorded were: Glena unipennaria, G. bipennaria, G. demissaria, G. brachia, Physocleora junctilinea, P. cariaria, Physocleora sp., Iridopsis fulvitincta, I. nigraria, Hymenomima amberia, Macaria regulata, Ischnopteris inornata, Prochoerodes onustaria, Prochoerodes sp., Thyrinteina arnobia, Nematocampa sp., Melanolophia sp., Isochromodes sp., Semaeopus lunifera, S. vizaria. The Hymenoptera were: Microcharops peronata, Charops sp., Metopius sp., Hyposoter sp., Mesochorus sp. (Ichneumonidae); Aleiodes sp., Meteorus sp., Glyptapanteles sp., Protapanteles sp. (Braconidae); Euplectrus sp. (Eulophidae). The Diptera, Tachinidae were: Winthemia sp. (Winthemiini) and a Blondeliini species. The period of higher occurrence of Geometridae larvae and lowest rate of parasitism was during the end of rainy season and the beginning of the dry season.
\end{abstract}

KEYWORDS. Bionomics; Brazil; cerrado; São Paulo.

RESUMO. Larvas de Geometridae (Lepidoptera) e seus parasitóides, associadas a Erythroxylum microphyllum St.-Hilaire (Erythroxylaceae). O trabalho foi realizado em área de cerrado, em São Carlos, São Paulo, Brasil. Foram realizadas amostragens quinzenais de junho/1999 a junho/2000, por meio de guarda-chuva-entomológico. Foram registradas 22 espécies de Geometridae pertencentes a 14 gêneros; quanto aos parasitóides, 11 gêneros de Hymenoptera e dois de Diptera, Tachinidae. A ocorrência de Cyclomia mopsaria (Geometridae) foi de 83\%. Outros Geometridae registrados foram: Glena unipennaria, G. bipennaria, G. demissaria, G. brachia, Physocleora junctilinea, P. cariaria, Physocleora sp., Iridopsis fulvitincta, I. nigraria, Hymenomima amberia, Macaria regulata, Ischnopteris inornata., Prochoerodes onustaria, Prochoerodes sp., Thyrinteina arnobia, Nematocampa sp., Melanolophia sp., Isochromodes sp., Semaeopus lunifera, S. vizaria. Os Hymenoptera foram: Microcharops peronata, Charops sp., Metopius sp., Hyposoter sp., Mesochorus sp. (Ichneumonidae); Aleiodes sp., Meteorus sp., Glyptapanteles sp., Protapanteles sp. (Braconidae); Euplectrus sp. (Eulophidae). Os Diptera, Tachinidae foram: Winthemia sp. (Winthemiini) e uma espécie de Blondeliini. O período de maior ocorrência de larvas de Geometridae e baixa taxa de parasitismo foi ao final da estação chuvosa e início da estação seca.

PALAVRAS-CHAVE. Bionomia; Brasil; cerrado; São Paulo.

Estudos que relacionam Lepidoptera, suas plantas hospedeiras e seus parasitóides são raros nos neotrópicos. Diniz \& Morais (1995, 1997), Diniz et al. (1999, 2001), Milhomen et al. (1997) e Pinheiro et al. (1997) referiram-se a lepidópteros em plantas de cerrado no Brasil central; Braga et al. (2001) aos Geometridae e seus parasitóides, associados a Piperaceae em área de mata semidecídua em São Carlos, SP; Scherrer et al. (1997) a Iridopsis sp. (Geometridae) parasitada por Braconidae, em cerrado.

Foram estudados os Geometridae associados a Erythroxylum microphyllum St.-Hilaire (Erythroxylaceae), com o objetivo de conhecer sua diversidade sobre essa planta, bem como seus eventuais parasitóides. O trabalho foi realizado no campus da Universidade Federal de São Carlos, município de São Carlos, São Paulo, entre as coordenadas $21^{\circ} 58^{\prime}$ e $22^{\circ}$ $00^{\prime}$ de latitude sul e $47^{\circ} 51^{\prime}$ ' e $47^{\circ} 52^{\prime}$ de longitude oeste. As coletas foram realizadas em área de cerrado de 124,68 hectares, na qual foi percorrido um caminho de 3.000 metros com periodicidade quinzenal, entre junho de 1999 e junho de 2000. Cerca de 50 plantas hospedeiras de porte arbustivo foram sacudidas sobre guarda-chuva entomológico. Em laboratório as larvas foram mantidas em recipientes plásticos com ramos da planta hospedeira, até o completo desenvolvimento, assim como as pupas até a eclosão dos adultos. Todos os exemplares, inclusive parasitóides, foram preservados segundo as técnicas usuais e depositados na coleção entomológica do 
Departamento de Ecologia e Biologia Evolutiva da Universidade Federal de São Carlos.

Foram obtidas 1560 larvas, as quais incluíram 14 gêneros e 22 espécies de Geometridae (Tabela I). Ocorreram 204 larvas parasitadas por Hymenoptera (Eulophidae, Ichneumonidae e Braconidae) (Tabela II) e duas larvas parasitadas por Diptera (Tachinidae).

A maior ocorrência de larvas foi de janeiro a maio de 2000. Cyclomia mopsaria Guenée, [1858] representou 83\% das larvas coletadas; larvas dos demais gêneros ocorreram em pequena porcentagem e outros gêneros somaram 2\% (Tabela I). Diniz \& Morais (1995, 1997), Milhomen et al. (1997) e Diniz et al. (2001) registraram C. mopsaria em Erythroxylum tortuosum Martius, E. suberosum St.-Hilaire e E. deciduum St.-Hilaire no cerrado de Brasília, DF. Dados sobre estágios imaturos e bionomia de C. mopsaria estão em Marconato \& Dias (2004).

Dos 13 gêneros de parasitóides que ocorreram, o mais freqüente foi Microcharops Roman, 1910 (Ichneumonidae) (53\%), seguido de Euplectrus Westwood, 1832 (Eulophidae) (21\%). Os parasitóides foram mais freqüentes na época de baixa pluviosidade, especialmente no mês de agosto. O período com menor taxa de parasitismo correspondeu àquele de maior abundância do hospedeiro; muitas larvas de Geometridae foram registradas em abril e maio. Segundo Barbosa et al. (2001) plantas com maior número de folhas ocultam o hospedeiro e aumentam a área de busca do parasitóide. Durante a estação seca as folhas de E. microphyllum estão em processo de senescência, o que reduz a qualidade nutricional da planta para a larva, além de torná-la mais vulnerável por perda de abrigo contra predadores e parasitóides.

Hymenoptera, Chalcidoidea (Eulophidae, Eulophinae). Euplectrus sp.: Foram registradas 46 larvas de C. mopsaria parasitadas, cujas larvas são gregárias e se alojam sobre o dorso da larva hospedeira. O número de larvas parasitóides variou de acordo com o instar em que se encontrava o hospedeiro; larvas hospedeiras de instares iniciais podem apresentar apenas uma larva de Euplectrus sp.; larvas de quinto instar podem ter até 12 larvas parasitóides. As pupas do parasitóide ficam sob os despojos da larva hospedeira, que passam a ser uma proteção; estas ficam envoltas por casulos rudimentares, dispostos em fileira única sob a larva hospedeira. Segundo Yamamoto et al. (1998) larvas de Lepidoptera são impedidas de realizar ecdise devido à injeção de uma substância pelas fêmeas de Euplectrus spp. durante a oviposição; foi observada uma larva de $C$. mopsaria que sofreu ecdise do terceiro para o quarto instar, eliminando os parasitóides junto com a exúvia, porém, essa larva não mais se alimentou e pereceu. Em outra larva do mesmo hospedeiro houve multiparasitismo por Euplectrus sp. e Microcharops peronata (Cameron, 1911) (Ichneumonidae); as larvas de Euplectrus sp. não chegaram a empupar e a de $M$. peronata teceu casulo sob os despojos da larva hospedeira, mas não houve eclosão do parasitóide adulto (Fig. 4).

Hymenoptera, Ichneumonoidea (Ichneumonidae).
Campopleginae. Charops sp.: Foram obtidos quatro exemplares hospedados por larvas de C. mopsaria (dois indivíduos), Glena sp. e Iridopsis fulvitincta (Warren, 1897). Hábito solitário. O parasitóide eclodiu de um casulo oval, alongado, com manchas claras sobre fundo escuro. Os despojos da larva hospedeira não ficaram aderidos ao casulo do parasitóide; o casulo foi preso à planta por um fio de seda em posição mediana; o parasitóide eclodiu por uma abertura apical ao casulo.

Hyposoter sp.: Dois indivíduos em C. mopsaria e um em I. fulvitincta. Hábito solitário. A larva do parasitóide deixou a larva hospedeira após ter se alimentado de todo o seu conteúdo, restando apenas o tegumento translúcido. O parasitóide empupou em um casulo oval, castanho-claro, com faixas transversais pretas, preso à planta por sua face lateral; estágio pupal de 11 a 12 dias.

Microcharops peronata (Cameron, 1911): Ocorreram 115 casos de parasitismo por M. peronata em C. mopsaria (113 exemplares), Macaria regulata (Fabricius, 1775) e Prochoerodes sp. Parasitóide de hábito solitário, suas larvas foram observadas saindo das larvas hospedeiras quando estas se encontravam no quinto instar, exceto por um registro no quarto instar. Ao final do ciclo de parasitismo, as larvas hospedeiras ficaram dilatadas na metade posterior do corpo e a coloração tornou-se pálida; a larva do parasitóide saiu pela região anterior da larva hospedeira, ventralmente, próximo ao primeiro par de pernas torácicas. A empupação ocorreu sob o tegumento vazio do hospedeiro, que ficou aderido ao casulo

Tabela I. Geometridae com larvas registradas em Erythroxylum microphyllum (Erythroxylaceae), em São Carlos, São Paulo, e suas respectivas porcentagens de ocorrência; asteriscos indicam espécies com ocorrência inferior a $1 \%$.

\section{ENNOMINAE}

Cyclomia mopsaria Guenée, [1858]

Glena bippenaria (Guenée, [1858])

Glena brachia Rindge, 1967

Glena demissaria (Walker, 1860)

Glena unipennaria (Guenée, [1858])

Herbita renipuncta (Warren, 1895)

Hymenomima amberia (Schaus, 1901)

Iridopsis fulvitincta (Warren, 1897)

Iridopsis nigraria (Jones, 1921)

Ischnopteris inornata Pitkin, 2005

Isochromodes sp.

Macaria regulata (Fabricius, 1775)

Melanolophia sp.

Nematocampa sp.

Physocleora cariaria (Schaus, 1897)

Physocleora junctilinea (Warren, 1901)

Physocleora sp.

Prochoerodes onustaria (Hübner, 1832) $3 \% \quad 3 \%$

Prochoerodes sp.

Thyrinteina arnobia (Stoll, 1782)

STERRHINAE

Semaeopus lunifera (Warren, 1897)

Semaeopus vizaria (Schaus, 1901)
$4 \%$

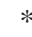

$3 \%$

$2 \%$

$*$

$*$

$2 \%$

$*$

$*$

$1 \%$

$*$

$*$ 

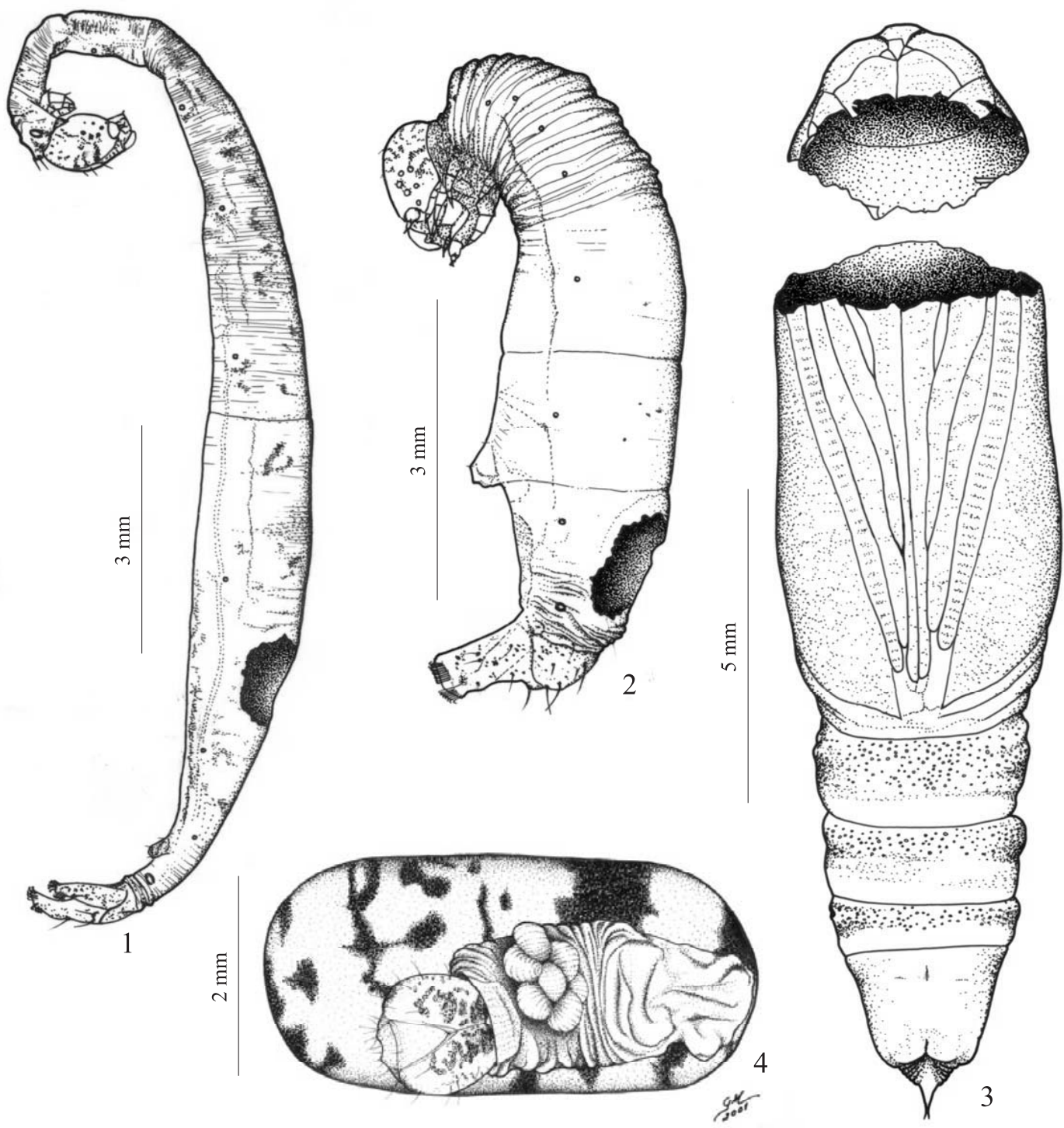

Figs. 1-4. 1, Parasitismo por Aleiodes sp. (Braconidae) em Cyclomia mopsaria (Geometridae), larva hospedeira mumificada, com abertura dorsal por onde eclodiu o parasitóide; 2, idem, larva com corpo menos distendido. 3, Parasitismo por Metopius sp. (Ichneumonidae) em Glena sp. (Geometridae) (pupa do hospedeiro com abertura por onde eclodiu o parasitóide); 4, Multiparasitismo por Euplectrus sp. (Eulophidae) (larvas ectoparasitóides) e Microcharops peronata (casulo), em larva de Cyclomia mopsaria (Geometridae).

do parasitóide. O casulo é cilíndrico, com extremidades arredondadas, de coloração castanho-clara amarelada, com manchas castanho-escuras e brancas; o orifício de saída do parasitóide adulto foi feito em uma das extremidades do casulo, o qual ficou aderido à planta e sobre ele, a cápsula cefálica e restos de tegumento do hospedeiro. Estágio pupal de 6 a 8 dias.

Mesochorinae. Mesochorus sp.: Oito exemplares obtidos; são hiperparasitóides solitários e foram registrados sobre Campopleginae, cujo hospedeiro primário foi C. mopsaria. $\mathrm{O}$ período entre a empupação do hospedeiro secundário e a eclosão do parasitóide foi de 9 a 11 dias.

Metopiinae. Metopius sp.: Hábito solitário. Um exemplar eclodiu de pupa de Glena sp. (Fig. 3), esta obtida de larva coletada na natureza. Estágio pupal de 21 dias.

Braconidae. Meteorinae. Meteorus sp.: Foram obtidos 16 exemplares em larvas de C. mopsaria. A larva parasitóide desenvolveu-se na porção posterior do hospedeiro e saiu por um orificio lateral em A6 ou A7, em larvas de quarto ou quinto instar; empupou em casulo amarelo-acastanhado, preso à planta por fio de seda de 2 a $3 \mathrm{~cm}$; o casulo se prende pela extremidade menos afilada e o parasitóide eclode pela extremidade oposta.

Microgastrinae. Glyptapanteles sp.: Seis exemplares hospedados por C. mopsaria (4 exemplares), Glena sp. e Physocleora sp. A larva (solitária) do parasitóide saiu da larva 
Tabela II. Parasitóides de Geometridae e seus respectivos hospedeiros registrados em Erythroxylum microphyllum (Erythroxylaceae), em São Carlos, São Paulo.

\begin{tabular}{|c|c|}
\hline HOSPEDEIRO & PARASITÓIDES \\
\hline \multirow[t]{14}{*}{ Cyclomia mopsaria } & Ichneumonidae \\
\hline & Charops sp. \\
\hline & Hyposoter sp. \\
\hline & Mesochorus sp. \\
\hline & Microcharops peronata \\
\hline & Braconidae \\
\hline & Aleiodes sp. \\
\hline & Glyptapanteles sp. \\
\hline & Meteorus sp. \\
\hline & Eulophidae \\
\hline & Euplectrus sp. \\
\hline & Tachinidae \\
\hline & Winthemiini, Winthemia sp. \\
\hline & Blondeliini \\
\hline \multirow[t]{5}{*}{ Glena sp. } & Ichneumonidae \\
\hline & Metopius sp. \\
\hline & Charops sp. \\
\hline & Braconidae \\
\hline & Glyptapanteles sp. \\
\hline \multirow[t]{2}{*}{ Hymenomima amberia } & Braconidae \\
\hline & Protapanteles sp. \\
\hline \multirow[t]{4}{*}{ Macaria regulata } & Ichneumonidae \\
\hline & Microcharops peronata \\
\hline & Braconidae \\
\hline & Protapanteles sp. \\
\hline \multirow[t]{3}{*}{ Iridopsis fulvitincta } & Ichneumonidae \\
\hline & Charops sp. \\
\hline & Hyposoter sp. \\
\hline \multirow{3}{*}{ Physocleora sp. } & Braconidae \\
\hline & Aleiodes sp. \\
\hline & Glyptapanteles sp. \\
\hline \multirow[t]{2}{*}{ Prochoerodes sp. } & Ichneumonidae \\
\hline & Microcharops peronata \\
\hline
\end{tabular}

hospedeira por um orificio em A8; teceu casulo castanho ou branco, envolto frouxamente por fios de seda. Estágio pupal de 5 a 7 dias.

Protapanteles sp.: Dois exemplares, hospedados por Hymenomima amberia (Schaus, 1901) e Macaria regulata (Fabricius, 1775). A larva (solitária) do parasitóide saiu do hospedeiro por um orifício em A8 e teceu um casulo branco.

Rogadinae. Aleiodes sp.: Três exemplares obtidos de larvas de C. mopsaria (dois exemplares) (Figs. 1 e 2) e Physocleora sp. Parasitóide solitário, empupou dentro da larva hospedeira, mumificando-a. A eclosão do parasitóide ocorreu por uma abertura dorsal na extremidade posterior do hospedeiro mumificado. Shaw (2006) ilustrou as larvas mumificadas de algumas espécies de Geometridae parasitadas por Aleiodes spp.

Recebido em 09/03/2007; aceito em 21/11/2007
Diptera. Tachinidae. Ocorreram duas espécies de Goniinae, pertencentes a Blondeliini e Winthemiini (Winthemia sp.); cada um desses parasitóides teve apenas um registro sobre $C$. mopsaria durante o período de coleta. As larvas parasitóides eclodiram da pupa do hospedeiro e empuparam no substrato.

Agradecimentos. À CAPES (Coordenação de Aperfeiçoamento de Pessoal de Nível Superior), CNPq (Conselho Nacional de Desenvolvimento Científico e Tecnológico) e FAPESP (Fundação de Amparo à Pesquisa do Estado de São Paulo) por auxílios concedidos; ao Dr. Waldir Mantovani pela identificação da planta hospedeira; ao Dr. José Henrique Guimarães pela identificação dos dípteros.

\section{REFERÊNCIAS}

Barbosa, P; A. E. Segarra; P. Gross; A. Caldas; K. Ahlstrom; R. W. Carlson; D. C. Ferguson; E. E. Grissell; R. W. Hodges; P. M. Marsh; R. W. Poole; M. E. Schauff; S. R. Shaw; J. B. Whitfield \& N. E. Woodley. 2001. Differential parasitism of macrolepidopteran herbivores on two deciduous trees species. Ecology 82: 698-704.

Braga, S. M. P.; M. M. Dias \& A. M. Penteado-Dias. 2001. Aspectos bionômicos de Eois tegularia (Guenée) e Eois glauculata (Walker) ( Lepidoptera, Geometridae, Larentiinae) e seus parasitóides. Revista Brasileira de Zoologia 18: 837-840.

Diniz, I. R. \& H. C. Morais. 1995. Larvas de Lepidoptera e suas plantas hospedeiras em um cerrado de Brasília, DF, Brasil. Revista Brasileira de Entomologia 39: 755-770.

Diniz, I. R. \& H. C. Morais. 1997. Lepidopteran caterpillar fauna of cerrado host plants. Biodiversity and Conservation 6: 817836.

Diniz, I. R.; H. C. Morais; A. M. F. Botelho; F. Venturoli \& B. C. Cabral. 1999. Lepidopteran caterpillar fauna on lactiferous host plants in the central Brazilian cerrado. Revista Brasileira de Biologia 59: $627-635$.

Diniz, I. R.; H. C. Morais \& A. J. A. Camargo. 2001. Host plantas of lepidopteran caterpillars in the cerrado of the Distrito Federal, Brazil. Revista Brasileira de Entomologia 45: 107-122.

Marconato, G. \& M. M. Dias. 2004. Estágios imaturos e bionomia de Cyclomia mopsaria Guenée (Lepidoptera, Geometridae). Revista Brasileira de Entomologia 48: 1-8.

Milhomen, M. S.; H. C. Morais; I. R. Diniz \& J. D. Hay. 1997. Espécies de lagartas em Erythroxylum spp. (Erythroxylaceae) em um cerrado de Brasília, p. 107-111. In: Leite, L. L. \& C. H. Saito (Orgs). Contribuição ao conhecimento ecológico do cerrado. Universidade de Brasília. 326 p.

Pinheiro, F.; H. C. Morais \& I. R. Diniz. 1997. Composição de herbívoros em plantas hospedeiras com látex: Lepidoptera em Kielmeyera spp. (Guttiferae), p. 101-106. In: Leite, L. L. \& C. H. Saito (Orgs). Contribuição ao conhecimento ecológico do cerrado. Universidade de Brasília. 326 p.

Shaw, S. R. 2006. Aleiodes wasps of eastern forests: a guide to parasitoids and associated mummified caterpillars. Morgantown, Forest Health Technology Enterprise Team - 2006 08 , iv $+121 \mathrm{p}$.

Scherrer, S.; I. R. Diniz \& H. C. Morais. 1997. Caracterização da fauna de parasitóides (Hymenoptera) de lagartas, no cerrado de Brasília, p. 131-134. In: Leite, L. L. \& C. H. Saito (Orgs). Contribuição ao conhecimento ecológico do cerrado. Universidade de Brasília. $326 \mathrm{p}$.

Yamamoto, A. C.; A. K. Doetzer \& L. A. Foerster. 1998. Efeito da temperatura no desenvolvimento de Euplectrus ronnai (Brèthes) (Hymenoptera, Eulophidae) parasitando lagartas de Pseudaletia sequax Franclemont (Lepidoptera, Noctuidae) e impacto do parasitismo no consumo alimentar do hospedeiro. Acta Biológica Paranaense 27: 85-95. 